\title{
High Spin Topologically Massive Gravity
}

\author{
Bin Chen ${ }^{1,2,3 *}$ Jiang Long ${ }^{1 \dagger}$ \\ ${ }^{1}$ Department of Physics, and State Key Laboratory of Nuclear Physics and Technology, \\ Peking University, Beijing 100871, P.R. China \\ ${ }^{2}$ Center for High Energy Physics, Peking University, Beijing 100871, P.R. China \\ ${ }^{3}$ Kavli Institute for Theoretical Physics China, CAS, Beijing 100190, P.R. China
}

October 11, 2018

\begin{abstract}
We propose an action to describe high spin topologically massive gravity with a negative cosmological constant. With the frame-like fields and spin connections being combined into two gauge fields, the action includes two gauge field Chern-Simons actions with different levels, and also a linear term proportional to the difference of the gauge field strengths. Such linear term play the role of imposing the torsion free conditions for high spin fields. We discuss the gauge symmetry of this action and study the fluctuations around the $\mathrm{AdS}_{3}$ vacuum. We show how to relate the the fluctuations of the gauge field to the Frondal fields, using the gauge symmetry. For the gauge group $S L(n, R) \times$ $S L(n, R)$, we find that the fluctuations of all high spin fields up to spin $n$ satisfy third order differential equations, and hence there generically exist massive traceless and trace mode for every spin.
\end{abstract}

\footnotetext{
*Email:bchen01@pku.edu.cn

${ }^{\dagger}$ Email:1j301@pku.edu.cn
} 


\section{Introduction}

In the past decade, the theory of high spin fields has drawn much attention, especially in AdS/CFT correspondence. It has been known for a long time that even though the free high spin fields could be defined in flat and curved spacetime[1, the interacting high spin theory could only be defined in Anti-de Sitter (AdS) and de Sitter (dS) spacetime[2, 3, 4]. Especially in $D \geq 4$ spacetime, once an interacting higher spin field is considered, all the spin fields up to spin $\infty$ have to be taken into account, with additional compensator fields. Nevertheless, it has been conjectured that Vasiliev's minimal bosonic high spin field theory in $\mathrm{AdS}_{4}$ is holographically dual to the singlet sector of three-dimensional $O(N)$ model in the large $\mathrm{N}$ and free field limit [5]. The nontrivial checks on the three-point correlation functions have been done few years ago $[$.

Since the summer of last year, the study of the high spin $\mathrm{AdS}_{3}$ gravity has been developing very quickly. In this case, the compensator fields are not needed and more importantly, it is possible to define an interacting high spin gravity theory with finite spin truncation [7, 8]. In a remarkable paper [8, the authors showed that the high spin $\mathrm{AdS}_{3}$ gravity theory with spin up to $n$ could be written as a Chern-Simons gravity theory with $S L(n, R) \times$ $S L(n, R)$ gauge group 11 . By imposing appropriate boundary condition and gauge choice, they showed that the asymptotic symmetry group of high spin $\mathrm{AdS}_{3}$ gravity is a classical $W_{n}$ symmetry with the same central charge in the pure $\mathrm{AdS}_{3}$ gravity found in 13 . This indicates that the high spin $\mathrm{AdS}_{3}$ gravity is holographically dual to a conformal field theory with $W_{n}$ symmetry. Similar observation has been made in [7] starting from the $n \rightarrow$ $\infty$ theory. For the further developments in high spin $\mathrm{AdS}_{3}$ gravity and its dual, see [16, 17, 18, 19, 20, 21, 22, 23, 24, 25, 28, 29]. For the study of black holes with high spin hair, see [14, 15, 26, 27].

For pure gravity and high spin fields in three dimensions, there is no local degree of freedom, even though there do exist boundary degrees of freedom at asymptotic infinity, as shown in the existence of BTZ black hole and the black holes with high spin hair. One possible way to induce local dynamical degree of freedom without bringing in ghost is to introduce the gravitational Chern-Simons term and its cousin for high spin fields. The resulting topologically massive gravity has been studied for almost thirty years since its proposal[30, 31]. It was found generically such a theory has an extra local massive mode, but it suffers from either the instability of the vacuum or negative energy of the black hole. However, few years ago, it was found that at a critical point, the theory could be well-defined, especially after imposing Brown-Henneaux boundary condition. At the critical point,

\footnotetext{
${ }^{1}$ For original study on the relation between $\mathrm{AdS}_{3}$ gravity and Chern-Simons theory, see 9 , 10. For the earlier study of the high spin field theory in $\mathrm{AdS}_{3}$ in the framework of Chern-Simons gravity, see [11, 12].
} 
the theory is called chiral gravity [32, 33], as the local massive mode becomes degenerate with the left massless mode and the only degree of freedom is a massless boundary graviton. It was conjectured that chiral gravity is dual to a chiral CFT with only right-mover. It is remarkable that even at the chiral point, there is actually a logarithmic mode, which carries negative energy 34, 35. It has been suggested that there exists a logarithmic CFT dual to the TMG at the chiral point [36, 37, 38, and the chiral gravity could only be a consistently truncated subsector of the whole theory after imposing the Brown-Henneaux boundary condition [33].

It would be interesting to investigate if there exist some kind of generalization of gravitational Chern-Simons term for the higher spin field and how the high spin field coupled to the topologically massive gravity. Actually, in [39, a Chern-Simons-like term for spin-3 field has been proposed in flat spacetime. And very recently, the high spin topologically massive gravity has been studied. In [40], the action of spin-3 topologically massive gravity has been proposed in the first order formulation. The action could be rewritten as the sum of two Chern-Simons gauge field action with different levels, and two extra terms imposing the torsion free conditions. The extra terms are essential to impose the torsion free condition and moreover leads to a massive mode for the fluctuations around the $\mathrm{AdS}_{3}$ vacuum. The action not only includes the gravitational Chern-Simons term for the graviton, but also the Chern-Simons-like term for the spin-3 field in $\mathrm{AdS}_{3}$ [41]. In [40], it was shown that at the critical point, the traceless part of spin-3 fluctuation has only massless boundary right-mover, keeping the chiral nature of the theory 2. The trace part was later investigated in [41]. However, in order to study the other high spin fields, the extra terms have to be introduced to impose the torsion free condition for every spin field. This incumber the study of higher spin topologically massive gravity. Another disadvantage in using extra torsion-imposing term is that the action is not gauge invariant, since the Chern-Simons parts are written in terms of the gauge fields while the extra terms are linear in torsions.

In this paper, we propose an action for high spin topologically massive gravity. In this new action, all of the extra terms imposing the torsion free conditions for the high spin fields could be encoded into a single term, which is linearly proportional to the difference of the field strength of two gauge potential. Starting from this action, we discuss the gauge symmetry of the action and investigate the fluctuations around the $\mathrm{AdS}_{3}$ vacuum. With the re-emergent linearized gauge symmetry in the background of $\mathrm{AdS}_{3}$, we show how to write vierbein as symmetric doubly traceless field and its trace. Moreover, we manage to study all the high spin fluctuations and obtain their

\footnotetext{
${ }^{2}$ This is actually true only when some generalized Brown-Henneaux boundary condition has been imposed such that the logarithmic mode carrying negative energy could be truncated consistently. The existence of the logarithmic modes at the critical points was found in both the traceless part [40] and the trace part 41].
} 
equations of motion, which are third order differential equations. These equations reproduce exactly the existing results in the literature [42]. It is remarkable that in [41, 42], the equations of motion of high spin fluctuations around $\mathrm{AdS}_{3}$ were derived from the linearized action for high-spin Frondal fields with Chern-Simons-like terms, where the coefficients of the ChernSimons-like terms for the spin higher than 3 were set by demanding the chiral point of high spin fields coincide with the one of gravity. In this paper, our analysis is based on the first order action of the frame-like fields and spinconnections. We show how to relate the fluctuations of the frame-like fields to the Frondal fields, and derive the equations of motion of all higher spin fluctuations. Therefore the match of the equations of motion suggest that the Chern-Simons-like terms suggested in 42] are correct on one hand, and our theory is reasonable on the other hand. Certainly, our action includes not only the linearized action, but also the interactions among the graviton and other higher spin fields.

The remaining parts of the paper are organized as follows. In the next section, we introduce the action of high spin topologically massive gravity. In Sec. 3, we discuss the symmetry of the action. In Sec. 4, we discuss the fluctuations in $\mathrm{AdS}_{3}$ vacuum and obtain their equations of motion. We end with some discussions in Sec. 5. In the appendices, we include the convention on $S L(n, R)$ algebra, and some technical details.

\section{Action}

We start from the following action

$$
S_{T M G}=\frac{k}{4 \pi}\left[\left(1-\frac{1}{\mu l}\right) S_{C S}[A]-\left(1+\frac{1}{\mu l}\right) S_{C S}[\bar{A}]-\frac{1}{\mu} \int \operatorname{tr}(\beta \wedge(F-\bar{F}))\right] .
$$

where we have the Chern-Simons action as

$$
S_{C S}[A]=\int \operatorname{tr}\left(A \wedge d A+\frac{2}{3} A \wedge A \wedge A\right),
$$

the gauge curvature

$$
F=d A+A \wedge A, \quad \bar{F}=d \bar{A}+\bar{A} \wedge \bar{A} .
$$

and one-form Lagrangian multiplier $\beta$. The gauge field $A, \bar{A}$ and the Lagrangian multiplier $\beta$ are in the adjoint representation of the corresponding group. The gauge field $A$ and $\bar{A}$ are related to the frame-like fields and spin connection as 3

$$
A=\omega+e, \quad \bar{A}=\omega-e,
$$

There are several remarks on the action (1):

\footnotetext{
${ }^{3}$ From now on we denote the $A d S$ radius $l=1$.
} 
1. Firstly, the action is defined without explicit dependence on the gauge group. In other words, as an action on gauge fields, it make sense for any group which can be interpreted as higher spin field coupled to gravity. In this paper we consider the group $S L(n, R) \times S L(n, R)$ and discuss the fields of spin from 2 to $n$;

2. Secondly, when $\mu \rightarrow \infty$, it reduces to the action of the well-known high spin $\mathrm{AdS}_{3}$ gravity which describes a tower of higher spin fields from spin 3 to spin $n$ coupled to gravity provided the gauge group is chosen to be $S L(n, R) \times S L(n, R)[8$;

3. Thirdly, when $\beta \neq 0$, the last term is a Lagrangian multiplier. The imposed condition $F=\bar{F}$ looks strange, but it is nothing but torsionfree condition. If the gauge group is chosen as $S L(2, R) \times S L(2, R)$, then the condition reduces to the torsion-free condition on the spin connection and the first-order action (11) is equivalent to the famous topologically massive gravity [30, 31]. If the gauge group is chosen to be $S L(3, R) \times S L(3, R)$, the conditions once again give the torsionfree conditions on both spin-2 and spin-3 connection, and the action defines the so-called spin-3 topologically massive gravity [40];

4. For general gauge group $S L(n, R) \times S L(n, R)$, the condition $F=\bar{F}$ encodes all the torsion-free conditions on the spin-connections, where the torsions are the same as the ones in usual higher spin $\mathrm{AdS}_{3}$ gravity, which could be defined along the line in [8]. In this case, the action describe all the high spin fields coupled to topological massive gravity. In the action, there are not only topologically Chern-Simons term for graviton, but also the similar parity-breaking Chern-Simons terms for higher spin fields. The theory will be called by us as high spin topologically massive gravity;

5. Finally, the action is defined in terms of the gauge potential, without the torsion appearing explicitly. As a result, the equations of motion could be given in a concise form

$$
\begin{array}{r}
\left(1-\frac{1}{\mu}\right) F-\frac{1}{2 \mu}(d \beta+\beta \wedge A+A \wedge \beta)=0 \\
\left(1+\frac{1}{\mu}\right) \bar{F}-\frac{1}{2 \mu}(d \beta+\beta \wedge \bar{A}+\bar{A} \wedge \beta)=0 \\
F=\bar{F} .
\end{array}
$$

These equations are easier to deal with. In particular when we investigate the fluctuations around the $\mathrm{AdS}_{3}$ vacuum, the analysis is quite simple, even if we consider an arbitrary spin $n$ fluctuation. 


\section{Symmetry}

In this section we study the symmetry of the action which is essential for us to interpret it as a higher spin generalization of topologically massive gravity. Let us start from the case $\beta=0$. In this case, the action is invariant under the gauge transformation

$$
\delta A=d \lambda+[A, \lambda], \quad \delta \bar{A}=d \bar{\lambda}+[\bar{A}, \bar{\lambda}] .
$$

Another way to represent this gauge transformation is to introduce $\Lambda$ and $\tilde{\Lambda}$ by the relation

$$
\lambda=\Lambda+\tilde{\Lambda}, \quad \bar{\lambda}=\Lambda-\tilde{\Lambda},
$$

then

$$
\delta_{\Lambda} A=d \Lambda+[A, \Lambda], \quad \delta_{\Lambda} \bar{A}=d \Lambda+[\bar{A}, \Lambda]
$$

and

$$
\delta_{\tilde{\Lambda}} A=d \tilde{\Lambda}+[A, \tilde{\Lambda}], \quad \delta_{\tilde{\Lambda}} \bar{A}=-d \tilde{\Lambda}-[\bar{A}, \tilde{\Lambda}] .
$$

$\delta_{\Lambda}$ induces the usual Lorentz transformation while $\delta_{\tilde{\Lambda}}$ is related to the gauge transformations of physical fields.

Next we turn to the case $\beta \neq 0$. We observe that in the action the term linear in $\beta$ breaks the previous gauge symmetry since $F$ and $\bar{F}$ changes as

$$
F \rightarrow F+[F, \lambda], \quad \bar{F} \rightarrow \bar{F}+[\bar{F}, \bar{\lambda}] .
$$

Nevertheless, the Lorentz transformation is preserved. Actually, under the transformation

$$
\delta_{\Lambda} A=d \Lambda+[A, \Lambda], \quad \delta_{\Lambda} \bar{A}=d \Lambda+[\bar{A}, \Lambda], \quad \delta_{\Lambda} \beta=[\beta, \Lambda]
$$

the action is invariant. On the other hand, the gauge symmetry involving $\bar{\Lambda}$ is broken. Namely, the imposition of the torsion free condition breaks half of the gauge symmetries. This seems bad since the broken gauge symmetry is important for us to define the gauge transformations of the physical fields. However,this is not a serious problem for three reasons. The first reason is that the symmetry is broken only off-shell. The equation of motion tells us that $[F, \beta]=[\bar{F}, \beta]=0$ hence the symmetry is preserved on shell. The second reason is there is nothing wrong to break the symmetry off-shell by hand in field theory. In other words, if we ignore the gauge symmetry which is missing in our action, we just define a tower of higher rank field coupled to gravity. The third but most important reason is that although the half gauge symmetry is broken off-shell, if we focus on the fluctuations around $\mathrm{AdS}_{3}$ background, the broken gauge symmetry could be recovered at linearized level.

The emergence of the linearized gauge symmetry in the $\mathrm{AdS}_{3}$ vacuum can be shown as follows. It is easy to see that the $\mathrm{AdS}_{3}$ background with 
all higher spin fields vanishing is a solution of our theory. We take it as the vacuum. Considering the fluctuations around the vacuum, we expand the gauge fields as $A+a$ and $\bar{A}+\bar{a}$, where we denote the background field as $A$ and $\bar{A}$. Since in the vacuum $\beta=0$, we use $\beta$ to denote the fluctuations in the following discussion. Then we expand the original action in the $\mathrm{AdS}_{3}$ vacuum to quadratic order of the fluctuations and have

$$
S_{(2)}=\frac{k}{4 \pi}\left(S[a]-S[\bar{a}]+S_{\beta}\right)
$$

where

$$
\begin{gathered}
S[a]=\left(1-\frac{1}{\mu}\right) \int \operatorname{tr}((d a+A \wedge a+a \wedge A) \wedge a) \\
S[\bar{a}]=\left(1+\frac{1}{\mu}\right) \int \operatorname{tr}((d \bar{a}+\bar{A} \wedge \bar{a}+\bar{a} \wedge \bar{A}) \wedge \bar{a}), \\
S_{\beta}=-\frac{1}{\mu} \int \operatorname{tr}(\beta \wedge[(d a+A \wedge a+a \wedge A)-(d \bar{a}+\bar{A} \wedge \bar{a}+\bar{a} \wedge \bar{A})]) .
\end{gathered}
$$

Note that it is important to take the fluctuations $\beta$ into account as it induces extra massive degrees of freedom, as we will show later. We can show that the action is invariant under the following gauge transformations

$$
\delta_{\Lambda} a=d \Lambda+[A, \Lambda], \quad \delta_{\Lambda} \bar{a}=d \Lambda+[\bar{A}, \Lambda], \quad \delta_{\Lambda} \beta=0
$$

and

$$
\delta_{\tilde{\Lambda}} a=d \tilde{\Lambda}+[A, \tilde{\Lambda}], \quad \delta_{\tilde{\Lambda}} \bar{a}=-d \tilde{\Lambda}-[\bar{A}, \tilde{\Lambda}], \quad \delta_{\tilde{\Lambda}} \beta=0 .
$$

Note that the second gauge symmetry has been broken in the original action but it re-emerges in the background $\mathrm{AdS}_{3}$. This owes to the fact that in the $\mathrm{AdS}_{3}$ background $F=\bar{F}=0$.

Due to the re-emergence of the linearized gauge symmetry generated by $\tilde{\Lambda}$, we can define the spin-s fluctuation gauge fields as

$$
h_{\nu, \nu_{1} \cdots \nu_{s-1}}=\bar{e}_{\nu_{1}}^{a_{1}} \cdots \bar{e}_{\nu_{s-1}}^{a_{s-1}} e_{\nu a_{1} \cdots a_{s-1}}
$$

where $\bar{e}$ is $A d S_{3}$ background vierbein and we have chosen a general $S L(n, R) \times$ $S L(n, R)$ bases following [8]. The explicit form of the bases is given in Appendix A. After a Lorentz transformation we can set $h_{\nu, \nu_{1} \cdots \nu_{s-1}}$ field to a symmetric field

$$
\Phi_{\nu \nu_{1} \cdots \nu_{s-1}}=\frac{1}{s} \bar{e}_{\left(\nu_{1}\right.}^{a_{1}} \cdots \bar{e}_{\nu_{s-1}}^{a_{s-1}} e_{\nu) a_{1} \cdots a_{s-1}}
$$

which has a gauge symmetry generated by $\xi_{\nu_{1} \cdots \nu_{s-1}}=\bar{e}_{\nu_{1}}^{a_{1}} \cdots \bar{e}_{\nu_{s-1}}^{a_{s-1}} \tilde{\Lambda}_{a_{1} \cdots a_{s-1}}$

$$
\delta_{\xi} \Phi_{\nu \nu_{1} \cdots \nu_{s-1}}=\nabla_{(\nu} \xi_{\left.\nu_{1} \cdots \nu_{s-1}\right)} \text {. }
$$


From the definition of $\xi_{\nu_{1} \cdots \nu_{s-1}}$, we know that it is symmetric and traceless since $\tilde{\Lambda}$ is symmetric and traceless. Also, the traceless condition of $e_{\nu}^{a_{1} \cdots a_{s-1}}$ induces the double traceless condition of $\Phi_{\nu \nu_{1} \cdots \nu_{s-1}}$.

Since the procedure of symmetrization from $h$ field to $\Phi$ field is a key point in our discussion of the fluctuations, we would like to clarify this procedure more clearly. This has also been discussed in [3] in the flat spacetime background. Note that in our first order formulation, the Lorentz transformation becomes a gauge transformation generated by $\Lambda$. When we go back to the physical fields of higher spin $s$, we need to choose a gauge to avoid this redundant degree of freedom. $\Lambda$ can be parameterized as

$$
\Lambda=\sum_{s=2}^{s=n} \Lambda^{a_{1} \cdots a_{s-1}} T_{a_{1} \cdots a_{s-1}}
$$

where $\Lambda^{a_{1} \cdots a_{s-1}}$ is symmetric and traceless and the $S L(n, R)$ generators $T_{a_{1} \cdots a_{s-1}}$ are defined in Appendix A. When one goes from $h$ to $\Phi$, it is necessary to choose a gauge to eliminate the redundant degree of freedom generated by these $\Lambda^{a_{1} \cdots a_{s-1}}$. This is always possible since $h_{\nu, \nu_{1} \cdots \nu_{s-1}}$ has $3(2 s-1)$ independent components while $\Phi_{\nu \nu_{1} \cdots \nu_{s-1}}$ has $2(2 s-1)$ components. Their difference $(2 s-1)$, is simply the number of independent components of symmetric traceless $\Lambda^{a_{1} \cdots a_{s-1}}$. We can make a decomposition of $h$ schematically as

$$
h \sim \Phi+\Lambda .
$$

The precise decomposition depends on their special properties, which we list them as follows

$$
\begin{gathered}
h_{\left(\nu, \nu_{1} \cdots \nu_{s-1}\right)}=s \Phi_{\nu \nu_{1} \nu_{s-1}}, \quad h_{\nu, \sigma \nu_{1} \cdots \nu_{s-3}}^{\sigma}=0 \\
\Phi^{\nu \sigma \sigma \nu_{1} \cdots \nu_{s-4}}=0 \\
\Theta_{\left(\nu, \nu_{1} \cdots \nu_{s-1}\right)}=0, \quad \Theta^{\nu}{ }_{\nu \nu_{2} \cdots \nu_{s-1}}=0, \quad \Theta_{\nu, \sigma \nu_{3} \cdots \nu_{s-1}}^{\sigma}=0 .
\end{gathered}
$$

Here $\Theta$ is defined as

$$
\Theta_{\nu, \nu_{1} \cdots \nu_{s-1}}=\epsilon_{\left(\nu_{1}\right.}^{\kappa \sigma} \bar{e}_{\mid \nu \kappa} \Lambda_{\left.\sigma \mid \nu_{2} \cdots \nu_{s-1}\right)},
$$

and appears in the Lorentz transformation of $h_{\nu \nu_{1} \cdots \nu_{s-1}}$. Then $h$ can be expressed as

$$
\begin{aligned}
h_{\nu, \nu_{1} \cdots \nu_{s-1}}= & \Phi_{\nu \nu_{1} \cdots \nu_{s-1}}+\Theta_{\nu, \nu_{1} \cdots \nu_{s-1}}+\frac{s-2}{2(s-1)} g_{\nu\left(\nu_{1}\right.} \Phi_{\left.\nu_{2} \cdots \nu_{s-1}\right)}^{\prime} \\
& -\frac{1}{s-1} g_{\left(\nu_{1} \nu_{2}\right.} \Phi_{\left.|\nu| \nu_{3} \cdots \nu_{s-1}\right)}^{\prime} .
\end{aligned}
$$

where we use prime to denote taking the trace part of the corresponding fields. We also use the convention that the symmetrization symbol denotes 
the minimal number of terms it encloses and without any normalization. This expression satisfies all the properties of $h$ and $\Phi$. Note that the naive decomposition of $h=\Phi+\Lambda$ is wrong since it violates the properties of $h$ and $\Phi$. In the next section, we will deal with the fluctuations around $A d S_{3}$ background and the decomposition (29) is important for our discussion.

\section{Fluctuations around $\mathrm{AdS}_{3}$ vacuum}

From the quadratical action of the fluctuations around $A d S_{3}$ vacuum we can derive their equations of motion

$$
\begin{aligned}
G(d a+a \wedge A+A \wedge a) & =d \beta+\beta \wedge A+A \wedge \beta \\
\bar{G}(d \bar{a}+\bar{a} \wedge \bar{A}+\bar{A} \wedge \bar{a}) & =d \beta+\beta \wedge \bar{A}+\bar{A} \wedge \beta \\
d a+a \wedge A+A \wedge a & =d \bar{a}+\bar{a} \wedge \bar{A}+\bar{A} \wedge \bar{a}
\end{aligned}
$$

where we have defined $G=2(\mu-1), \bar{G}=2(\mu+1)$. We find that in the $A d S_{3}$ background, this linearized equations describe free fluctuations of spin 2 to spin $n$ once we choose the basis of $S L(n, R)$ in the appendix A. The interaction between different spin is neglected since they are higher order terms.

When $\beta=0$, we use the previous decomposition of $h_{\nu \nu_{1} \cdots \nu_{s-1}}$ and choose a gauge that $\Theta_{\nu \nu_{1} \cdots \nu_{s-1}}=0$, then we can derive the Fronsdal equation [1]

$$
\begin{aligned}
\mathcal{F}_{\nu_{1} \cdots \nu_{s}} \equiv \square \Phi_{\nu_{1} \cdots \nu_{s}} & -\nabla_{\left(\nu_{1} \mid\right.} \nabla^{\sigma} \Phi_{\left.\sigma \mid \nu_{2} \cdots \nu_{s}\right)}+\frac{1}{2} \nabla_{\left(\nu_{1}\right.} \nabla_{\nu_{2}} \Phi_{\left.\nu_{3} \cdots \nu_{s}\right)}^{\prime} \\
& -\left(s^{2}-3 s\right) \Phi_{\nu_{1} \cdots \nu_{s}}-2 g_{\left(\nu_{1} \nu_{2}\right.} \Phi_{\left.\nu_{3} \cdots \nu_{s}\right)}^{\prime}=0
\end{aligned}
$$

When $\beta \neq 0$, in principle we will get a third order differential equation for each spin $s$ field. Here we give the algorithm to derive this equation in our formulation. Note that this algorithm still holds when $\beta=0$.

Step 1: Decompose the veilbein fluctuations as

$$
\begin{aligned}
h_{\nu, \nu_{1} \cdots \nu_{s-1}}= & \left.\Phi_{\nu \nu_{1} \cdots \nu_{s-1}}+\Theta_{\nu, \nu_{1} \cdots \nu_{s-1}}+\frac{s-2}{2(s-1)} g_{\nu\left(\nu_{1}\right.} \Phi_{\left.\nu_{2} \cdots \nu_{s-1}\right)}^{\prime}\right) \\
& -\frac{1}{s-1} g_{\left(\nu_{1} \nu_{2}\right.} \Phi_{\left.|\nu| \nu_{3} \cdots \nu_{s-1}\right)}^{\prime} .
\end{aligned}
$$

Step 2: Define a set of quantities as

$$
\begin{gathered}
Q_{c, a_{1} \cdots a_{s-1}}=\epsilon_{c}^{\mu \nu} \nabla_{\mu} e_{\nu a_{1} \cdots a_{s-1}}, \quad X_{c, a_{1} \cdots a_{s-1}}=Q_{c, a_{1} \cdots a_{s-1}}-\frac{1}{s} g_{c\left(a_{1}\right.} Q_{\left.a_{2} \cdots a_{s-1}\right)}^{\prime}, \\
W_{c, a_{1} \cdots a_{s-1}}=\frac{1}{2} \epsilon_{c}^{\mu \nu} F_{\mu \nu a_{1} \cdots a_{s-1}}, \quad P_{c, a_{1} \cdots a_{s-1}}=W_{c, a_{1} \cdots a_{s-1}}-\frac{1}{s} g_{c\left(a_{1}\right.} W_{\left.a_{2} \cdots a_{s-1}\right)}^{\prime} \cdot(
\end{gathered}
$$


Step 3: Use the equations of motion to derive the following relations

$$
\begin{aligned}
& \omega_{c, a_{1} \cdots a_{s-1}}=X_{c, a_{1} \cdots a_{s-1}}-\frac{1}{s-1} X_{\left(c, a_{1} \cdots a_{s-1}\right)}, \\
& \beta_{c, a_{1} \cdots a_{s-1}}=2\left[P_{c, a_{1} \cdots a_{s-1}}-\frac{1}{s-1} P_{\left(c, a_{1} \cdots a_{s-1}\right)}\right] .
\end{aligned}
$$

Step 4: Derive the equation of motion of $\beta$

$$
\epsilon_{c}^{\rho \nu} \nabla_{\rho} \beta_{\nu a_{1} \cdots a_{s-1}}=2 \mu W_{c, a_{1} \cdots a_{s-1}} .
$$

After symmetrization, one gets

$$
\epsilon_{\left(a_{1} \mid\right.}^{\rho \nu} \nabla_{\rho} \beta_{\left.\nu \mid a_{2} \cdots a_{s}\right)}=2 \mu W_{\left(a_{1}, a_{2} \cdots a_{s}\right)} .
$$

Step 5: Choose a gauge $\Theta_{\nu \nu_{1} \cdots \nu_{s-1}}=0$ and substitute $e_{\nu, a_{1} \cdots a_{s-1}}$ in terms of $\Phi_{\nu a_{1} \cdots a_{s-1}}$.

The calculation is quite tedious. The explicit form of $W_{c, a_{1} \cdots a_{s-1}}$ and $\beta_{c, a_{1} \cdots a_{s-1}}$ are shown in Appendix B. For arbitrary spin $s \geq 2$, we finally obtain the equations of the physical fields

$$
\mathcal{F}_{a_{1} \cdots a_{s}}+\frac{1}{\mu s(s-1)} \epsilon_{\left(a_{1} \mid\right.}^{b c} \nabla_{b} \mathcal{F}_{\left.c \mid a_{2} \cdots a_{s}\right)}=0
$$

To discuss free equations of motion of the fluctuations, we only need to know the commutation relation between $\mathrm{SL}(2, \mathrm{R})$ generators and high spin generators. This allows us to obtain the equations of motion for arbitrary spin up to $n$ in our formulation.

It is remarkable that the above equations are exactly those appear in 41, 42. Note that in [41, the form of the Chern-Simons-like term for spin-3 field was determined by the gauge invariance, with the coefficient being fixed by compare with the Chern-Simons gravity action. And in [42], the higher spin generalization of such Chern-Simons-like term was proposed, by taking into account the gauge invariance and the chiral nature of the theory. Therefore, our result suggest that this kind of term can be completely determined from our action.

\section{Discussion}

In this paper, we studied the high spin topologically massive gravity in the $\mathrm{AdS}_{3}$ vacuum. We firstly proposed an action (11) to describe the high spin fields coupled to the topologically gravity. One remarkable feature of the action is that the term imposing torsion free conditions is written in terms of gauge field strength. There is great advantage to use this action:

- It makes the equations of motion easier to solve. One may just solve the equations of motions for the gauge fields and the Lagrangian multiplier, without paying special attention to the torsion free condition; 
- It makes the analysis of the fluctuations around the vacuum much easier. It allows us to analyze all the fluctuations from spin 2 up to $\operatorname{spin} n$;

We found that the imposition of the torsion free condition broke the gauge symmetry off-shell, but there is still on-shell gauge symmetry. Moreover, around the $\mathrm{AdS}_{3}$ vacuum, the quadratic action of the fluctuations are gauge invariant. This allows us to decompose the fluctuations using the gauge symmetry. We showed that in our theory, the spin $s$ field has a massive propagating mode for generic value of $\mu l$, satisfying a third order differential equation.

In this paper, we focused on the gauge group $S L(n, R) \times S L(n, R)$ and obtained a high spin topologically massive gravity with a negative cosmological constant. We may choose other gauge groups. One option is to consider a supergroup, which could lead to a supersymmetric theory. Another interesting option is to consider $S U(n) \times S U(n)$ group, which may describe a high spin topologically massive gravity on a Euclideanised $\mathrm{AdS}_{3}$.

We showed that in the $\mathrm{AdS}_{3}$ vacuum, the theory is well-defined with recovered gauge symmetry at the quadratic order. There are other possible vacua in the theory. Even with the vanishing high spin fields, the gravity sector allows the warped spacetime, which has been conjectured to be holographically dual to a conformal field theory 43 . In such warped spacetime, the gauge symmetry of the fluctuations needs to be investigated carefully. It is quite possible that the theory is still well-defined and thus provides a nontrivial example for high spin fields coupled to gravity beyond the maximally symmetry spaces. It would be very interesting to study these cases.

The action (11) is more tractable than other forms of action in terms of the torsion. It may allows us to classify all the solutions. Even for the pure topologically massive gravity, it is not clear if there exists asymptotical $\mathrm{AdS}_{3}$ solution, which is not an Einstein manifold. It would be interesting to address this issue in our framework. Moreover, it would be important to search for other asymptotically $\mathrm{AdS}_{3}$ solutions with nontrivial high spin hair.

\section{Acknowledgments}

We are indebted to Jun-bao Wu for many valuable discussions, and giving us a hardcopy of Ref. [3], which is crucial for the accomplishment of this project. The work was in part supported by NSFC Grant No. 10975005.

\section{Appendix A}

We choose the same convention as [8]. The generators of $S L(n, R)$ are chosen such that

$$
T_{a}=J_{a}
$$


form $S L(2, R)$ algebra

$$
\left[J_{a}, J_{b}\right]=\epsilon_{a b c} J^{c}
$$

and

$$
\left[J_{a}, T_{a_{1} \cdots a_{s-1}}\right]=\epsilon_{a\left(a_{1}\right.}^{m} T_{\left.a_{2} \cdots a_{s-1}\right) m}
$$

for $s>2$. The commutation relation between $T_{a_{1} \cdots a_{s-1}}$ for $s>2$ is irrelevant for our discussion.

\section{Appendix B}

In this section, we show the explicit form of $W_{c, a_{1} \cdots a_{s-1}}$ and $\beta_{c, a_{1} \cdots a_{s-1}}$ in terms of the totally symmetric and doubly traceless field $\Phi_{a_{1} \cdots a_{s}}$.

$$
\begin{aligned}
W_{c, a_{1} \cdots a_{s-1}}= & -\frac{1}{s-1} \square \Phi_{c a_{1} \cdots a_{s-1}}+\frac{s^{2}-3 s}{s-1} \Phi_{c a_{1} \cdots a_{s-1}}+\frac{1}{s-1} \nabla_{c} \nabla^{\sigma} \Phi_{\sigma a_{1} \cdots a_{s-1}} \\
& +\frac{1}{s-1} \nabla_{\left(a_{1} \mid\right.} \nabla^{\sigma} \Phi_{\left.\sigma c \mid a_{2} \cdots a_{s-1}\right)}-\frac{1}{s-1} g_{c\left(a_{1} \mid\right.} \nabla^{\sigma} \nabla^{\nu} \Phi_{\left.\sigma \nu \mid a_{2} \cdots a_{s-1}\right)} \\
& -\frac{(s-2)(2 s+1)}{2(s-1)} g_{c\left(a_{1}\right.} \Phi_{\left.a_{2} \cdots a_{s-1}\right)}^{\prime}+\frac{1}{s-1} g_{\left(a_{1} a_{2} \mid\right.} \Phi_{\left.c \mid a_{3} \cdots a_{s-1}\right)}^{\prime} \\
& \left.+\frac{1}{2(s-1)} g_{c\left(a_{1}\right.} \nabla_{a_{2} \mid} \nabla^{\sigma} \Phi_{\left.\sigma \mid a_{3} \cdots a_{s-1}\right)}^{\prime}-\frac{1}{2(s-1)} \nabla_{\left(a_{1}\right.} \nabla_{a_{2} \mid} \Phi_{\left.c \mid a_{3} \cdots a_{s-1}\right)}^{\prime}\right) \\
& +\frac{1}{s-1} g_{c\left(a_{1}\right.} \square \Phi_{\left.a_{2} \cdots a_{s-1}\right)}^{\prime}-\frac{1}{s-1} \nabla_{\left(a_{1} \mid\right.} \nabla_{c} \Phi_{\left.\mid a_{2} \cdots a_{s-1}\right)}^{\prime} .
\end{aligned}
$$

Note that when $\beta=0$, we find that $W_{c, a_{1} \cdots a_{s-1}}=0$. To get the Fronsdal equation, we need using this equation to eliminate the term related to $g_{c\left(a_{1}\right.} \square \Phi_{\left.a_{2} \cdots a_{s-1}\right)}^{\prime}$ and then taking its symmetrization. The $\beta$ is

$$
\begin{aligned}
& \frac{(s-1)^{2}}{2} \beta_{c, a_{1} \cdots a_{s-1}}=\square \Phi_{c a_{1} \cdots a_{s-1}}-\left(s^{2}-3 s\right) \Phi_{c a_{1} \cdots a_{s-1}}-\nabla_{c} \nabla^{\sigma} \Phi_{\sigma a_{1} \cdots a_{s-1}} \\
& \left.-\nabla_{\left(a_{1} \mid\right.} \nabla^{\sigma} \Phi_{\left.\sigma c \mid a_{2} \cdots a_{s-1}\right)}-\frac{s-3}{s} g_{c\left(a_{1} \mid\right.} \nabla^{\sigma} \nabla^{\rho} \Phi_{\left.\sigma \rho \mid a_{2} \cdots a_{s-1}\right)}+\frac{2}{s} g_{\left(a_{1} a_{2} \mid\right.} \nabla^{\sigma} \nabla^{\rho} \Phi_{\left.\sigma \rho c \mid a_{3} \cdots a_{s-1}\right)}\right) \\
& +\frac{s-3}{s} g_{c\left(a_{1}\right.} \square \Phi_{\left.a_{2} \cdots a_{s-1}\right)}^{\prime}-\frac{2}{s} g_{\left(a_{1} a_{2} \mid\right.} \square \Phi_{\left.c \mid a_{3} \cdots a_{s-1}\right)}^{\prime}-\frac{s^{2}-5 s+8}{2} g_{c\left(a_{1}\right.} \Phi_{\left.a_{2} \cdots a_{s-1}\right)}^{\prime} \\
& +(s-3) g_{\left(a_{1} a_{2} \mid\right.} \Phi_{\left.c \mid a_{3} \cdots a_{s-1}\right)}^{\prime}+\frac{s}{2} \nabla_{c} \nabla_{\left(a_{1}\right.} \Phi_{\left.a_{2} \cdots a_{s-1}\right)}^{\prime}-\frac{s-2}{2} \nabla_{\left(a_{1} \mid\right.} \nabla_{c} \Phi_{\left.\mid a_{2} \cdots a_{s-1}\right)}^{\prime} \\
& +\frac{1}{2} \nabla_{\left(a_{1}\right.} \nabla_{a_{2}} \Phi_{\left.a_{3} \cdots a_{s-1}\right) c}^{\prime}+\frac{s-3}{2 s} g_{c\left(a_{1}\right.} \nabla_{a_{2} \mid} \nabla^{\sigma} \Phi_{\left.\sigma \mid a_{3} \cdots a_{s-1}\right)}^{\prime} \\
& -\frac{1}{s} g_{\left(a_{1} a_{2} \mid\right.} \nabla_{c} \nabla^{\sigma} \Phi_{\left.\sigma \mid a_{3} \cdots a_{s-1}\right)}^{\prime}-\frac{1}{s} g_{\left(a_{1} a_{2}\right.} \nabla_{a_{3} \mid} \nabla^{\sigma} \Phi_{\left.\sigma c \mid a_{4} \cdots a_{s-1}\right)}^{\prime}
\end{aligned}
$$

\section{References}

[1] C. Fronsdal, "Massless fields with integer spin" Phys. Rev. D 18 (1978) 3624 . 
[2] C. Aragone and S. Deser, "Consistency Problems of Hypergravity," Phys. Lett. B 86, 161 (1979).

[3] M. A. Vasiliev, "gauge' form of description of massless fields with arbitrary spin. (In Russian)," Yad. Fiz. 32 (1980) 855 [Sov. J. Nucl. Phys. 32 (1980) 439].

[4] M. A. Vasiliev, "Free massless fields of arbitrary spin in the de sitter space and initial data for a higher spin superalgebra," Fortsch. Phys. 35, 741 (1987) [Yad. Fiz. 45, 1784 (1987)].

[5] I. R. Klebanov, A. M. Polyakov, "AdS dual of the critical $\mathrm{O}(\mathrm{N})$ vector model," Phys. Lett. B550, 213-219 (2002).

[6] S. Giombi, X. Yin, "Higher Spin Gauge Theory and Holography: The Three-Point Functions," JHEP 1009, 115 (2010). "Higher Spins in AdS and Twistorial Holography," JHEP 1104, 086 (2011)."On Higher Spin Gauge Theory and the Critical O(N) Model," arXiv:1105.4011.

[7] M. Henneaux and S. J. Rey, "Nonlinear $W_{\text {infinity }}$ as Asymptotic Symmetry of Three-Dimensional Higher Spin Anti-de Sitter Gravity," JHEP 1012, 007 (2010).

[8] A. Campoleoni, S. Fredenhagen, S. Pfenninger and S. Theisen, "Asymptotic symmetries of three-dimensional gravity coupled to higher-spin fields," JHEP 1011, 007 (2010).

[9] A. Achucarro, P. K. Townsend, "A Chern-Simons Action for ThreeDimensional anti-De Sitter Supergravity Theories," Phys. Lett. B180, 89 (1986).

[10] E. Witten, "(2+1)-Dimensional Gravity as an Exactly Soluble System," Nucl. Phys. B311, 46 (1988).

[11] M. P. Blencowe, "A Consistent Interacting Massless Higher Spin Field Theory In D = (2+1)," Class. Quant. Grav. 6, 443 (1989).

[12] E. Bergshoeff, M. P. Blencowe, K. S. Stelle, "Area Preserving Diffeomorphisms And Higher Spin Algebra," Commun. Math. Phys. 128, 213 (1990).

[13] J. D. Brown and M. Henneaux, "Central Charges in the Canonical Realization of Asymptotic Symmetries: An Example from Three-Dimensional Gravity," Commun. Math. Phys. 104, 207 (1986).

[14] M. Gutperle, P. Kraus, "Higher Spin Black Holes," JHEP 1105, 022 (2011). 
[15] M. Ammon, M. Gutperle, P. Kraus and E. Perlmutter, "Spacetime Geometry in Higher Spin Gravity," arXiv:1106.4788.

[16] M. R. Gaberdiel, R. Gopakumar, A. Saha, "Quantum $W$-symmetry in $A d S_{3}, "$ JHEP 1102, 004 (2011).

[17] M. R. Gaberdiel, R. Gopakumar, "An $A d S_{3}$ Dual for Minimal Model CFTs," Phys. Rev. D83, 066007 (2011).

[18] M. R. Gaberdiel, T. Hartman, "Symmetries of Holographic Minimal Models," JHEP 1105, 031 (2011).

[19] M. R. Gaberdiel, R. Gopakumar, T. Hartman, S. Raju, "Partition Functions of Holographic Minimal Models," arXiv:1106.1897.

[20] A. Castro, A. Lepage-Jutier and A. Maloney, "Higher Spin Theories in $\mathrm{AdS}_{3}$ and a Gravitational Exclusion Principle," JHEP 1101, 142 (2011).

[21] C. Ahn, "The Large N 't Hooft Limit of Coset Minimal Models," arXiv:1106.0351.

[22] C. M. Chang and X. Yin, "Higher Spin Gravity with Matter in $\mathrm{AdS}_{3}$ and Its CFT Dual," arXiv:1106.2580.

[23] D. Polyakov, "A String Model for AdS Gravity and Higher Spins," arXiv:1106.1558.

[24] A. Campoleoni, S. Fredenhagen, S. Pfenninger, "Asymptotic $\mathrm{W}$-symmetries in three-dimensional higher-spin gauge theories," arXiv:1107.0290 [hep-th]].

[25] A. Castro, T. Hartman, A. Maloney, "The Gravitational Exclusion Principle and Null States in Anti-de Sitter Space," Class. Quant. Grav. 28, 195012 (2011). arXiv:1107.5098 [hep-th]].

[26] P. Kraus, E. Perlmutter, "Partition functions of higher spin black holes and their CFT duals," arXiv:1108.2567 [hep-th]]

[27] A. Castro, E. Hijano, A. Lepage-Jutier, A. Maloney, "Black Holes and Singularity Resolution in Higher Spin Gravity," arXiv:1110.4117 [hepth]].

[28] E. A. Bergshoeff, M. Kovacevic, J. Rosseel, P. K. Townsend, Y. Yin, "A spin-4 analog of 3D massive gravity," arXiv:1109.0382 [hep-th]].

[29] H. Lu, K. -N. Shao, "Solutions of Free Higher Spins in AdS," arXiv:1110.1138 [hep-th]].

[30] S. Deser, R. Jackiw, S. Templeton, "Three-Dimensional Massive Gauge Theories," Phys. Rev. Lett. 48, 975-978 (1982). 
[31] S. Deser, R. Jackiw, S. Templeton, "Topologically Massive Gauge Theories," Annals Phys. 140, 372-411 (1982).

[32] W. Li, W. Song, A. Strominger, "Chiral Gravity in Three Dimensions," JHEP 0804, 082 (2008).

[33] A. Maloney, W. Song, A. Strominger, "Chiral Gravity, Log Gravity and Extremal CFT," Phys. Rev. D81, 064007 (2010).

[34] S. Carlip, S. Deser, A. Waldron, D. K. Wise, "Cosmological Topologically Massive Gravitons and Photons," Class. Quant. Grav. 26, 075008 (2009).

[35] S. Carlip, S. Deser, A. Waldron, D. K. Wise, "Topologically Massive AdS Gravity," Phys. Lett. B666, 272-276 (2008).

[36] D. Grumiller and N. Johansson, "Instability in cosmological topologically massive gravity at the chiral point," JHEP 0807, 134 (2008).

[37] K. Skenderis, M. Taylor and B. C. van Rees, "Topologically Massive Gravity and the AdS/CFT Correspondence," JHEP 0909, 045 (2009).

[38] M. R. Gaberdiel, D. Grumiller and D. Vassilevich, "Graviton 1-loop partition function for 3-dimensional massive gravity," JHEP 1011, 094 (2010).

[39] T. Damour, S. Deser, "'geometry' Of Spin 3 Gauge Theories," Annales Poincare Phys. Theor. 47, 277 (1987).

[40] Bin Chen, Jiang Long, Jun-bao Wu, "Spin-3 Topological Massive Gravity",arXiv:1106.5141 [hep-th]

[41] Arjun Bagchi, Shailesh Lal, Arunabha Saha, Bindusar Sahoo, "Topologically Massive Higher Spin Gravity", arXiv:1107.0915[hep-th]

[42] Arjun Bagchi, Shailesh Lal, Arunabha Saha, Bindusar Saho "One loop partition function for topologically massive higher spin gravity", arXiv:1107.2063 [hep-th]

[43] D. Anninos, W. Li, M. Padi, W. Song and A. Strominger, Warped $A d S_{3}$ black holes, arXiv:0807.3040]. 